UCRL-JC-128352

PREPRINT

\title{
Laser Conditioning Study of KDP on the Optical Sciences Laser Using Large Area Beams
}

\author{
M. Runkel, J. De Yoreo, W. Sell, D Milam
}

This paper was prepared for submittal to the 29th Annual Boulder Damage Symposium

Boulder, $\mathrm{CO}$

October 6-8, 1997

December 20, 1997

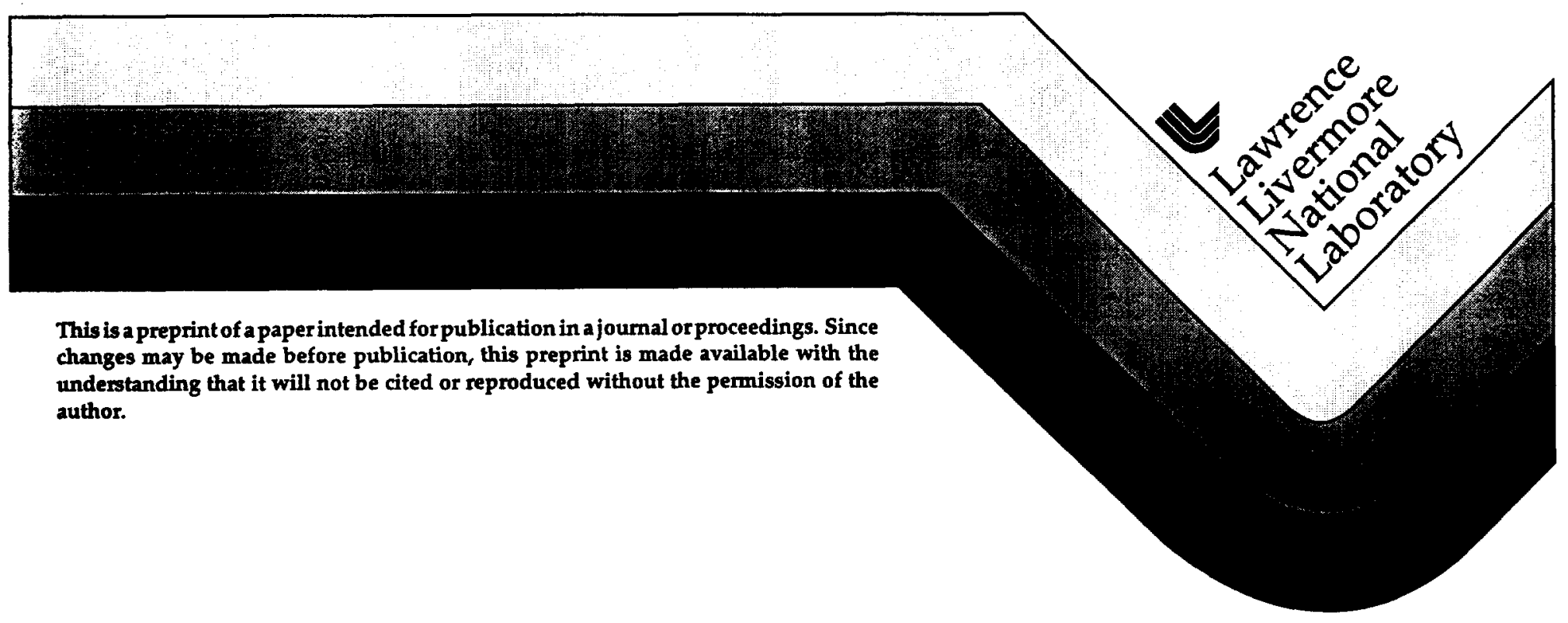




\section{DISCLAIMER}

This document was prepared as an account of work sponsored by an agency of the United States Government. Neither the United States Government nor the University of California nor any of their employees, makes any warranty, express or implied, or assumes any legal liability or responsibility for the accuracy, completeness, or usefulness of any information, apparatus, product, or process

disclosed, or represents that its use would not infringe privately owned rights. Reference herein to any specific commercial product, process, or service by trade name, trademark, manufacturer, or otherwise, does not necessarily constitute or imply its endorsement, recommendation, or favoring by the United States Government or the University of California. The views and opinions of authors expressed herein do not necessarily state or reflect those of the United States Government or the University of California, and shall not be used for advertising or product endorsement purposes. 


\title{
Laser conditioning study of KDP on the Optical Sciences Laser using large area beams
}

\author{
Mike Runkel, Jim DeYoreo, Walt Sell and David Milam \\ Lawrence Livermore National Laboratory \\ P.O. Box 808, L-250 \\ Livermore, CA 94550
}

\begin{abstract}
Considerable attention has been paid over the years to the problem of growing high purity $\mathrm{KDP}$ and $\mathrm{KD} * \mathrm{P}$ to meet damage threshold requirements on succeeding generations of inertial confinement fusion lasers at LLNL. While damage thresholds for these materials have increased over time, the current National Ignition Facility (NIF) maximum fluence requirement (redline) for $\mathrm{KD}^{* P}$ frequency triplers of $14.3 \mathrm{~J} / \mathrm{cm}^{2}$ at $351 \mathrm{~nm}, 3 \mathrm{~ns}$ has not been reached without laser (pre)conditioning. It is reasonable to assume that, despite the rapid increase in damage thresholds for rapidly grown crystals, -a program of large scale conditioning of the 192 NIF triplers will be required. Small area ramp $(R / 1)$ tests on single sites indicate that KDP damage thresholds can be raised on average up to $1.5 \mathrm{X}$ the unconditioned values. Unpublished LLNL $3 \omega$ raster conditioning studies on KDP, however, have not conclusively shown that off-line conditioning is feasible for $K D * P$. Consequently, investigating the feasibility of on-line conditioning of NIF triplers at $3 \omega$ has become a high priority for the KDP damage group at LLNL.
\end{abstract}

To investigate the feasibility of on-line conditioning we performed a series of experiments using the Optical Sciences Laser (OSL) on numerous samples of conventional and rapid growth KDP and KD*P. The experiment entailed exposing sites on each sample to a range of ramped shot (N/1) sequences starting at average fluences of $-2 \mathrm{~J} / \mathrm{cm}^{2}$ (in a $7 \mathrm{~mm}$ "top hat" beam @ $351 \mathrm{~nm}, 3 \mathrm{~ns}$ ) up to peak fluences of approximately $13 \mathrm{~J} / \mathrm{cm}^{2}$. Test results indicated that the most effective conditioning procedure entailed a 7-8 shot ramp starting at $2 \mathrm{~J} / \mathrm{cm}^{2}$ and ending at $12-13 \mathrm{~J} / \mathrm{cm}^{2}$. The pinpoint onset fluence for the $8 / 1$ tests was 1.4 times that of the unconditioned site. Damage evolution appears to be exponential as a function of increasing fluence. When damage occurs after conditioning however, pinpoint density evolution exhibits a greater slope than less conditioned sites. The overall reduction in the total pinpoint number can be as high as $300 \mathrm{X}$. Despite laser conditioning, the pinpoint onset for the samples considered is below the NIF redline fluence of $14.3 \mathrm{~J} / \mathrm{cm}^{2}$. In addition, the exponential pinpoint evolution curves indicate that damage levels at NIF redline fluences will be on the order of $10^{4}$ pinpoints $/ \mathrm{mm}^{3}$. This suggests that there will be significant damage in NIF triplers, however, substantial damage has not been observed in the large Beamlet tripler (conventionally grown KD*P) under similar exposure conditions. By applying the OSL damage evolution curves to model NIF THG output spatial profiles it is possible to show damage in NIF triplers will be slight, consisting of isolated clusters with a few pinpoints at high fluence portions of the beam. This prediction has been verified by scatter mapping the $37 \mathrm{~cm}$ Beamlet tripler crystal. These results will be discussed in a future memo.

These results indicate the feasibility of on-line conditioning for the NIF laser.

Keywords: KDP, DKDP, KD*P, bulk laser damage, laser conditioning

\section{INTRODUCTION}

The maximum $351 \mathrm{~nm}$ ( $3 \omega$ ) fluence specifications for Triple Harmonic Generator (THG) crystals to be used on the National Ignition Facility (NIF) have placed stringent demands on the laser damage performance of the $\left(D_{x} H_{1-x}\right)_{2} P O_{4}(K D * P, D K D P)$ crystals to be used as frequency converters. The current maximum fluence specification is $14.3 \mathrm{~J} / \mathrm{cm}^{2}$ at $351 \mathrm{~nm}$ in a $3 \mathrm{~ns}$ gaussian pulse. Extensive testing of KDP and $K D * P$ crystals at $L L N L{ }^{1,2}$ has shown that damage thresholds have increased with time, but not enough for even the best $\mathrm{KD}^{*} \mathrm{P}$ crystals to survive the NIF specification without some form of preconditioning. Our database reveals that the typical level of $3 \omega$ laser conditioning for both $\mathrm{KDP}$ and $\mathrm{KD} * \mathrm{P}$ is approximately $1.5 \mathrm{X}$. This data has been generated using LLNL's standard unconditioned $(\mathrm{S} / 1)$ and conditioned $(\mathrm{R} / 1)$ test procedures. While the $R / 1$ test procedure gives an overall level of conditioning it is unable to give information on when the maximum level of conditioning occurs, or whether there is a ramp rate dependence. A number of studies have also been undertaken at LLNL to examine $1064 \mathrm{~nm}(1 \omega)$ laser irradiation, thermal annealing and raster scanning as methods of 
conditioning KDP/KD*P to higher damage thresholds ${ }^{3-5}$. The results of these studies have been to show that 1) thermal annealing raises $1 \omega$ damage thresholds to $1 \omega$ laser conditioned levels but is not effective in increasing $3 \omega$ damage thresholds and 2) raster scanning at $1 \omega$ and $3 \omega$ is apparently not an effective conditioning method. The results of OSL testing described below, however, raise the issue of revisiting raster scanning as an effective means of off-line conditioning. There have also been other studies performed to investigate alternative methods for raising the damage thresholds of KDP. In 1992 Sun et. al. ${ }^{6}$ used $\mathrm{XeCl}(308 \mathrm{~nm}), 1 \omega \mathrm{Nd}: \mathrm{YAG}$ and a $\mathrm{CW} \mathrm{CO} \mathrm{CO}_{2}$ laser to pre-irradiate $\mathrm{KDP}$ crystals which were subsequently tested at 1.06 microns with a $10 \mathrm{~ns}$ pulse. They reported an average increase in the $1 \omega$ bulk damage threshold of $3.4 \mathrm{X}$ for $\mathrm{XeCl}$ pretreatment, $2 \mathrm{X}$ for $1 \omega \mathrm{Nd}$ :YAG pretreatment, and a drop of $1.6 \mathrm{X}$ due to $\mathrm{CO}_{2}$ irradiation. They did not perform any tests at $3 \omega$.

As a result of the importance of laser conditioning to the performance of NIF triplers and the lack of any studies pertaining to $3 \omega$ laser conditioning, we undertook a campaign designed to quantify a number of parameters associated with the $3 \omega$ laser conditioning process in KDP and KD*P. Our goals included determining 1) whether KDP/KD*P could be conditioned in a stepwise fashion using a large area beam (diameter greater than $1 \mathrm{~mm}$ ), 2) the degree of conditioning attainable depending on ramp rate, starting fluence and total number of shots, 3) correlating the test results between OSL and the ZEUS ${ }^{7}$ automated damage test system which uses a sub-millimeter beam and is the prototype QA tool for NIF crystals, 4) and ultimately whether on-line conditioning of KDP was feasible for the NIF project.

\section{TEST APPARATUS AND EXPERIMENTAL PROCEDURE}

\subsection{The OSL test layout.}

We used the Optical Sciences Laser (OSL) at LLNL to perform the tests. This system was chosen because it could deliver a 7 $\mathrm{mm}$ diameter, $351 \mathrm{~nm}$, near top hat beam (spatially and temporally) with enough fluence to damage our KDP and KD*P samples. In particular maximum fluences on the order of $20 \mathrm{~J} / \mathrm{cm}^{2}$ were attainable with a rep rate of 10 shots per day. Figure 1 shows the experimental layout. The output of the harmonic generator was image relayed through the spatial filter and appropriate focusing optics to the sample plane to provide the spatial top hat beam. Fixed attenuators were installed at two points in the beampath to provide energy control and a wedge was used to pick off portions of the beam for a standard set of beam diagnostics which included a camera (spatial profile), calorimeter (energy) and photodiode (temporal). The maximum beam modulation occurred near the outside edge and was as high as $\sim 2: 1$ (pk/av.). Aperture averaging the central $75 \%$ of the beam yielded a typical modulation of 1.3:1. The temporal pulsewidth was $3 \mathrm{~ns}$ and nearly top hat in nature. The characteristic parameters of the OSL laser are summarized in Table 1.

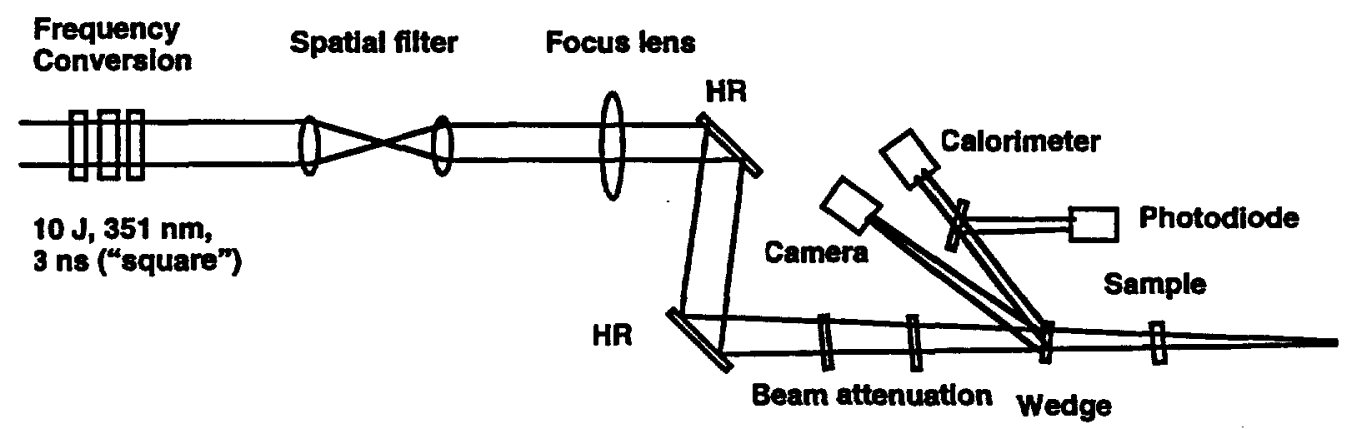

Figure 1. Schematic of the OSL beamline. The beam at the output of the frequency conversion crystals is image relayed to the sample plane. The energy was adjusted by a series of fixed attenuators to achieve maximum fluences of $17.5 \mathrm{~J} / \mathrm{cm}^{2}$ in 1.5 $\mathrm{J} / \mathrm{cm}^{2}$ steps. The wedge reflects $\sim 8 \%$ of the energy for the standard suite of beam diagnostics which is composed of camera, calorimeter and photodiode. The beam diameter at the sample plane was $-7.1 \mathrm{~cm}$. XY translation stages of 2 inch travel were used to translate the sample from site to site in the sample plane. The samples were set at 5-10 degrees angle of incidence to direct surface reflections into appropriate beam dumps.

Table 1. characteristic parameters of the OSL system 
Table 1. characteristic parameters of the OSL system

\begin{tabular}{|c|c|}
\hline Wavelength (nm) & 351 \\
\hline Maximum Energy (J) @ $351 \mathrm{~nm}$ & -10 \\
\hline Pulse duration (ns) @ 351 nm & 3 ns (rounded top hat) \\
\hline Pulse repetition frequency (shots/day) & 10 \\
\hline Beam diameter (mm) at sample plane & 7.1 \\
\hline Maximum average fluence $\left(\mathrm{J} / \mathrm{cm}^{2}\right)$ & -17.5 \\
\hline Peak beam modulation & $-2: 1$ \\
\hline Average beam modulation & $-1.3: 1$ \\
\hline
\end{tabular}

\subsection{ZEUS scatter diagnostic}

After the laser damage tests, the samples were mapped using a scatter diagnostic from the ZEUS system. As shown in Figure 2, a $30 \mathrm{~mW}$ HeNe laser and photomultiplier tube were used to illuminate and observe scatter from bulk damage pinpoint in the samples. Appropriate lenses, apertures and filters were used to image the bulk scatter at -45 degrees in the forward direction. The HeNe spot diameter at the sample was 300 microns and the samples were raster scanned across the beam in 50 micron steps using computer control. This choice of stepsize gave resolution comparable to the OSL spatial profile camera. The output voltage of the PMT was recorded by the computer during the scan. A variety of neutral density filters were used in the beamline to control saturation of the PMT voltage depending on the overall level of damage in the sample.

To obtain physically meaningful data from the raster scans, the PMT voltage was correlated to the density of scatter sites $\left(\mathrm{pp} / \mathrm{mm}^{3}\right)$. This was done by using $100 \mathrm{X}$ darkfield microscopy to count the number of damage sites (pinpoints) in the volume irradiated by the HeNe laser. This ultimately allowed us to develop a direct correlation between local damage density and local fluence in the beam and will be discussed below.

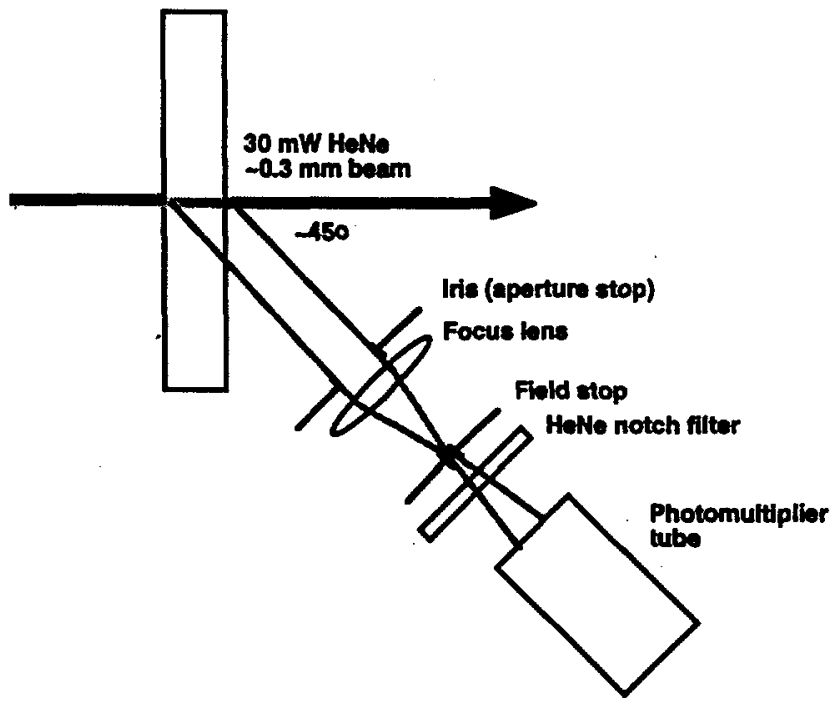

Figure 2. Schematic of the bulk scatter diagnostic used to measure damage on the OSL test samples after laser exposure.

\subsection{Test samples.}

The samples tested for the conditioning study included conventionally grown KDP and KD*P of Beamlet vintage (samples 328 and LL6-56) as well as the best KDP rapid growth samples made to date (214), and a typical rapidly grown KD*P crystal (DKDP11). Information on the samples is given in Table 2. All of the samples were plates cut perpendicular to the optic axis (z-plates) and were uncoated. 
Table 2. OSL sample test matrix. Fluence numbers refer to the percentage probability of failure and result from ZEUS automated damage tests? ${ }^{7}$. The associated damage probability curves are shown in Figure 3.

\begin{tabular}{|c|c|c|c|c|c|}
\hline Sample & Growth Method & $\begin{array}{l}\text { Conditioned } \\
P(F)=5 \%\end{array}$ & $\begin{array}{l}\text { ire fluence }(\mathrm{J} / \mathrm{cm} \\
P(F)=50 \% \pm \sigma\end{array}$ & $\begin{array}{l}3 \omega, 3 \text { ns }) \\
P(F)=100 \%\end{array}$ & Comments \\
\hline KDP 328 & Conventional & 13.1 & $25.9 \pm 4.8$ & 32.1 & 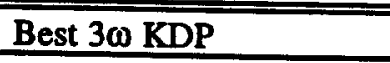 \\
\hline $\mathrm{KD} * \mathrm{P}$ LL6-56 & Conventional & 12.5 & $16.8 \pm 2.3$ & 22.0 & Best $3 \omega \mathrm{KD} * \mathrm{P}$ \\
\hline KDP 214 & Rapid & 11.9 & $16.3 \pm 2.7$ & 22.6 & Best $3 \omega$ fast grown KDP \\
\hline DKDP11 & Rapid & 7.1 & $10.9 \pm 2.5$ & 16.8 & Typical fast grown $\mathrm{KD}{ }^{*} \mathrm{P}$ \\
\hline
\end{tabular}

Samples 328 and LL6-56 represent the state-of-the-art in conventionally grown KDP and KD*P crystals. They exhibit the highest damage thresholds measured for KDP or KD*P respectively, regardless of growth technology. KDP 214 represents the current state-of-the-art in rapidly grown KDP. It was grown with ultra-high purity salts, continuous filtration and a stress relieving platform and is essentially identical in performance to LL6-56. DKDP11 represents a "typical" rapidly grown KD*P crystal grown with continuous filtration. The R/1 automated damage curves for these samples are shown in Figure 3.

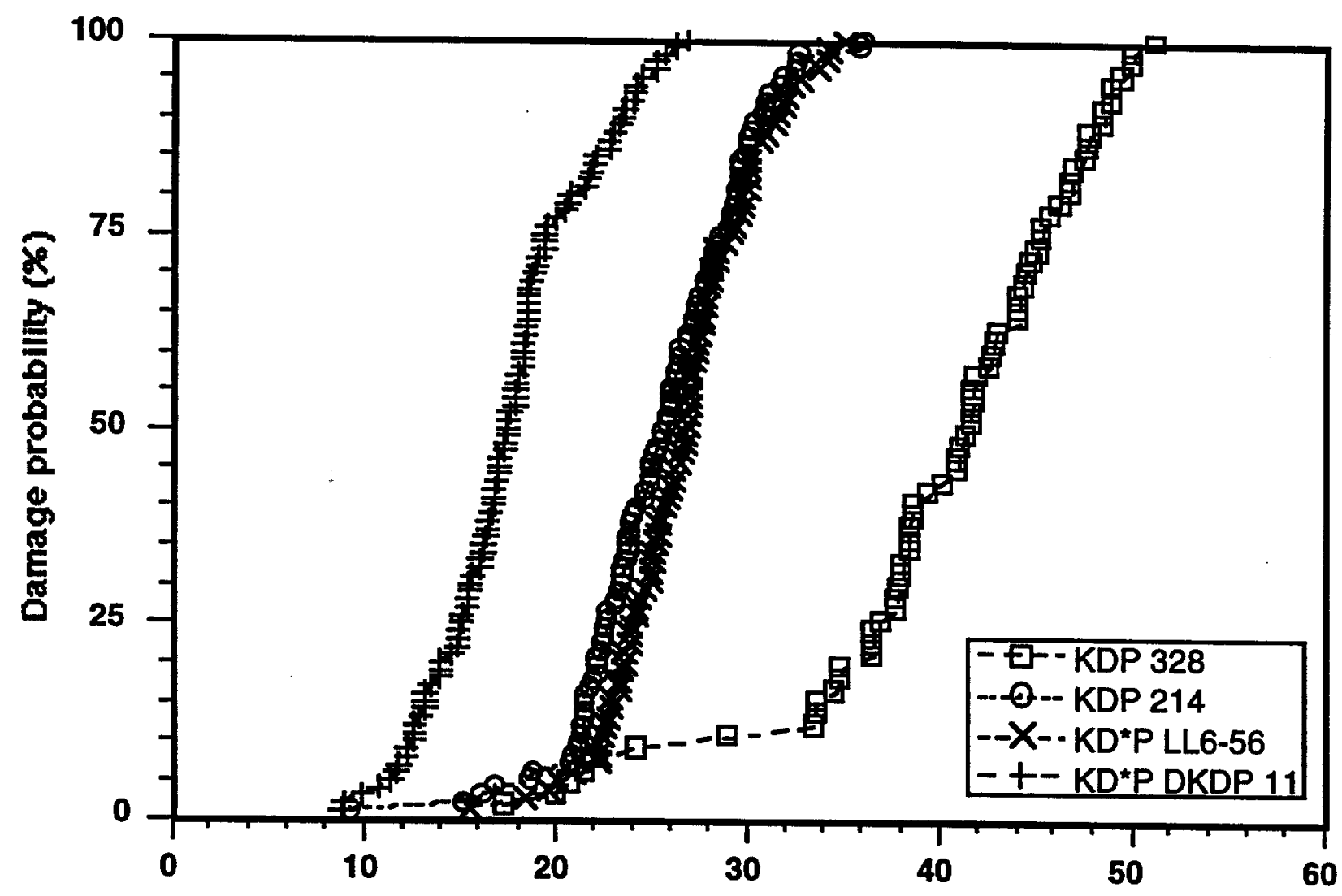

\section{Peak fluence (J/cm² $355 \mathrm{~nm}, 7.6 \mathrm{~ns})$}

Figure 3. Conditioned R/1 damage test curves from the ZEUS automated damage test system for the OSL test samples. Note that the plot is for a pulse duration of $7.6 \mathrm{~ns}$. $\tau^{0.5}$ scaling is used to convert between pulse durations.

\subsection{Experimental procedure}

Each sample was tested by first exposing a number of sites with a single shot (1/1) of increasing average fluence until damage was observed using visual inspection with backlighting against a dark background. Once this approximate damage threshold had been established the subsequent sites were exposed in ramps of equal fluence interval starting at an average fluence of $\sim 2$ $\mathrm{J} / \mathrm{cm}^{2}$. Testing continued on the sample until $4 / 1,8 / 1$ and $12 / 1$ ramps were performed to approximately the same final, 
average fluence. The sample was then taken to the ZEUS facility and scatter mapped. Figure 4 shows the series of ramped exposures for sample 214.

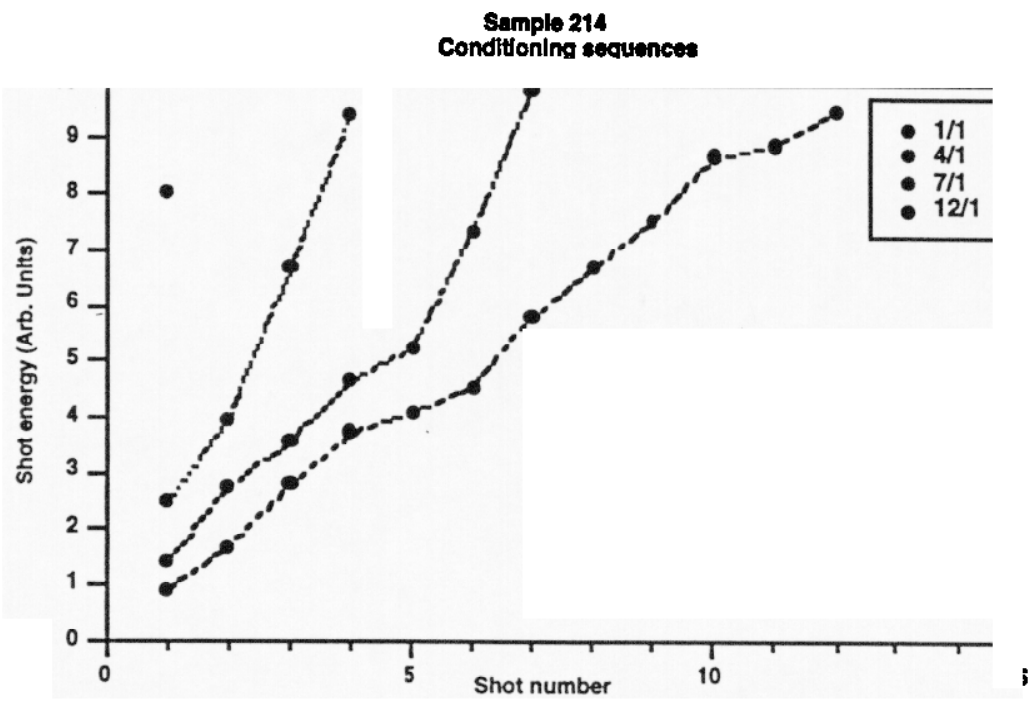

Figure 4. Ramped shot sequences for sample 214. After finding the approximate $1 / 1$ damage threshold, $4 / 1,8 / 1$ and $12 / 1$ ramps to the same approximate average fluence level were attempted. Due to fluctuations in the shot energy and the use of fixed attenuators, precise prediction of the average fluence on a given shot was not always possible. As a result the ramped sequences typically received higher average fluence on the final shot than for single shot exposures.

After mapping, the scatter data from a test site was imported into data analysis software which allowed images to be made. Scatter maps for sample 214 ramped exposures are shown in Figure 5.

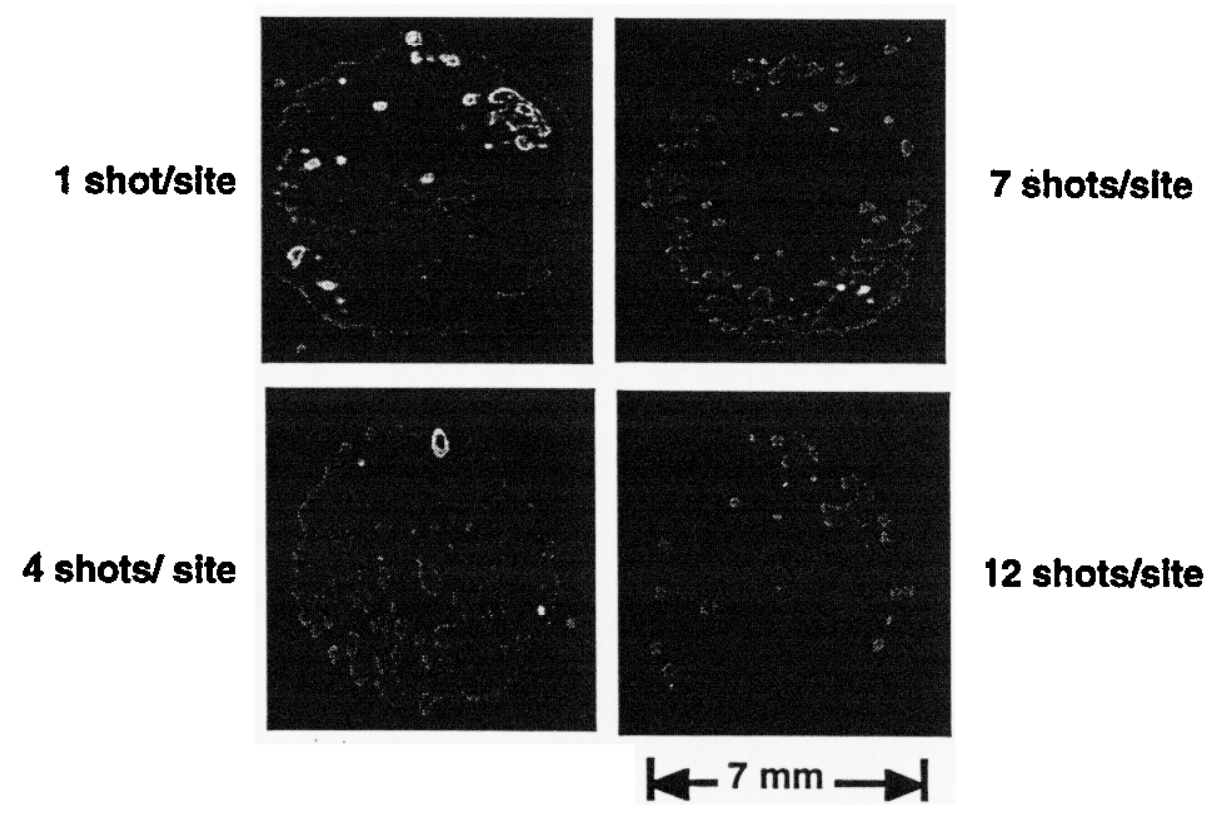

Figure 5. Scatter maps for sample 214. The $1 / 1$ sites shows more severe damage than the ramped sites despite being exposed to approximately $20 \%$ less average fluence. The reduction in damage severity continues to the $12 / 1$ case. 


\subsection{Damage morphology of sample 328}

The scatter maps show a dramatic reduction in the pinpoint density due to an increasing number of shots in the ramp. This behavior was repeated for all samples tested with the exception of sample 328. This sample exhibited no conditioning and did not damage until fluences near the OSL limit were reached. When damage did occur it was due to the high modulation portion of the beam where fluences were on the order of $\sim 30 \mathrm{~J} / \mathrm{cm}^{2}$ or higher. The morphology of this damage was significantly different from the other samples. It consisted of strings of pinpoints which repeated with regular frequency in the $\mathrm{XY}$ plane, as well as through the bulk of the sample. This damage morphology is compared to typical $\mathrm{KDP} / \mathrm{KD} * \mathrm{P}$ damage observed in the tests in Figure 6.
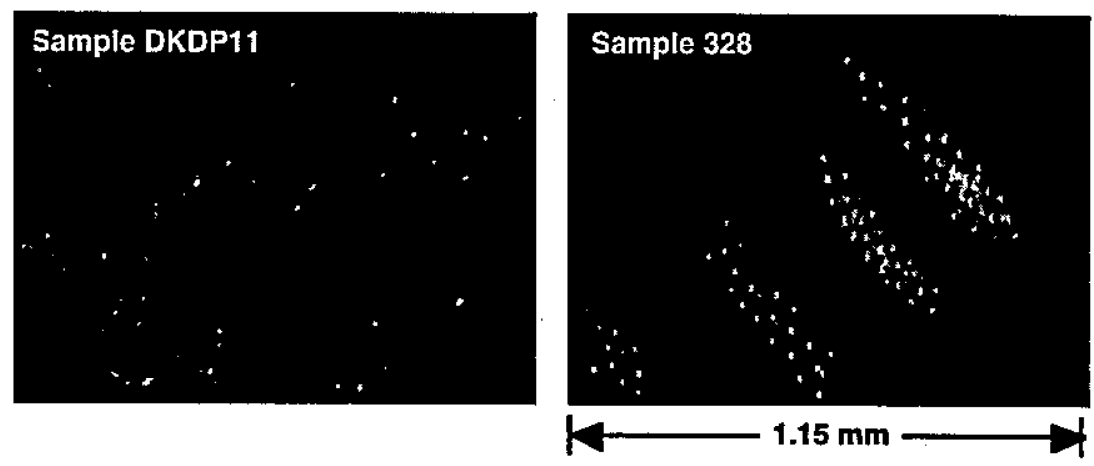

Figure 6. Darkfield micrographs of the randomly distributed pinpoints observed on DKDP11, 214 and LL6-56, and the periodic arrays of pinpoints observed on sample 328. The latter are probably due to self-focusing at $10 \mathrm{GW} / \mathrm{cm}^{2}$.

This type of damage has not been seen before on KDP at LLNL and we attribute it to small scale self-focusing of the $>10$ $\mathrm{GW} / \mathrm{cm}^{2}$ beam. We believe that this fluence indicates a threshold for activation of the intrinsic damage mechanism of ultra high purity material. Because of the extraordinary purity of this sample we also expect it to have very few extrinsic defects, and therefore a very large defect spacing. We hypothesize that laser conditioning is the removal or annealing of extrinsic defects, so observation of significant laser conditioning is not expected. In spite of the unusual and highly interesting damage morphology as well as our inability to laser condition sample 328 we have excluded discussion of it from the remainder of the paper.

\section{DATA REDUCTION AND ANALYSIS}

\subsection{Scatter diagnostic and beam fluence calibration}

In order to obtain physically meaningful results from the data it was necessary to calibrate the scatter diagnostic to the volume density of pinpoint damage ( $\mathrm{pp} / \mathrm{mm}^{3}$ ). This quantity was chosen because observations indicate that the number of damage sites increase with fluence but size does not change. Calibration of the scatter diagnostic involved straightforward counting of the pinpoints in the irradiated HeNe volume. After recording the scatter voltage from the PMT, the sample was rotated to a position allowing inspection of the damaged volume with $100 \mathrm{X}$ darkfield microscopy. For the case of low damage density a video monitor was used to view pinpoints which were counted manually as the field of view was scanned through the bulk of the crystal. A circular template corresponding to the 300 micron HeNe beam was used to exclude extraneous pinpoints on the monitor, after which the pinpoint density was calculated. For the case of severe damage, where the damage density was approximately uniform in any chosen field of view through the bulk, five photographs were taken at $\sim 200$ micron intervals through the sample. Again, a circular template was overlaid on the image and the pinpoints were counted. Statistics on the 5 slices were compiled and an average pinpoint density calculated. The data from this procedure was plotted versus the scatter voltage and a best fit line calculated to determine the response function of the diagnostic. Once the response function had been determined the scatter maps were converted from voltage to pinpoint density.

Raw data from the 8-bit spatial profile camera was converted to beam fluence by first calculating the effective area of each camera pixel, taking into account magnification in the diagnostic leg and the actual pixel dimension. The pixel's energy was 
obtained using the total number of counts on the pixel array and the total energy measured by the calorimeter for the shot. Once the scatter maps and fluence profiles for each shot had been obtained it was possible to make a direct comparison between the local fluence in the beam and damage.

To compare the OSL test results to ZEUS it was also necessary to calibrate the pinpoint density corresponding to detection of a single pinpoint as measured in a ZEUS automated test. This was done by considering the volume irradiated by ZEUS' 700 micron damage beam with peak fluence fluctuations of \pm 15 percent. We determined that the threshold sensitivity of the automated ZEUS system (ZEUS ADT) was $3 \mathrm{pp} / \mathrm{mm}^{3}$.

\subsection{Line out comparison of damage density and beam fluence}

As data analysis techniques were being developed, we determined that the fluence maps required different horizontal and vertical length scaling. This was attributed to astigmatism and rapid convergence of the OSL damage beam through the sample. To obtain proper scaling in both horizontal and vertical directions it was necessary to take lineouts through common features in the spatial profile and scatter maps. The fluence lineouts were then length scaled to give the best match to features in the corresponding scatter map lineout. Once horizontal and vertical length scale factors were determined the beam fluence was corrected and the pinpoint density versus fluence data for both lineouts was plotted. The lineout procedure is shown in Figure 7.

\section{Scatter Map of DKDP11-2, Site 2}

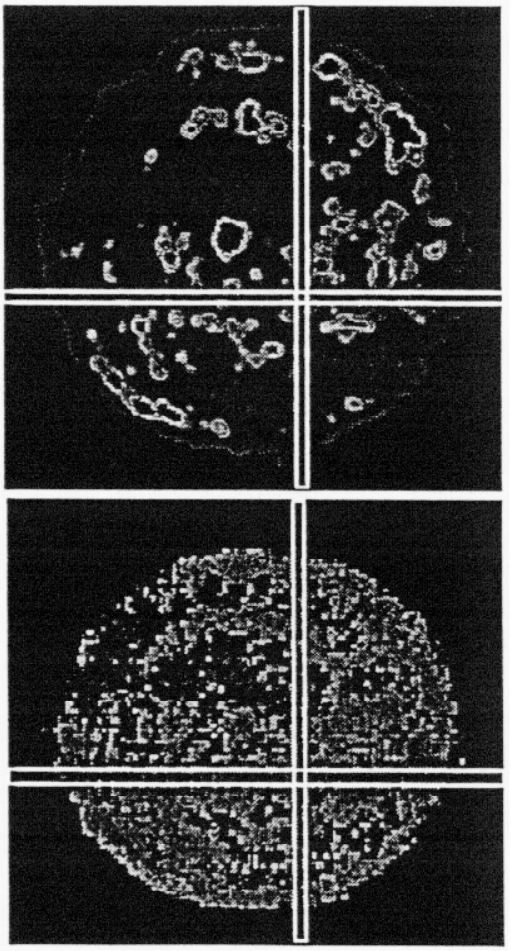

Spatial Profile
Point-by-point comparison of scatter to fluence allows the functional dependence $N(F)$ to be determined.

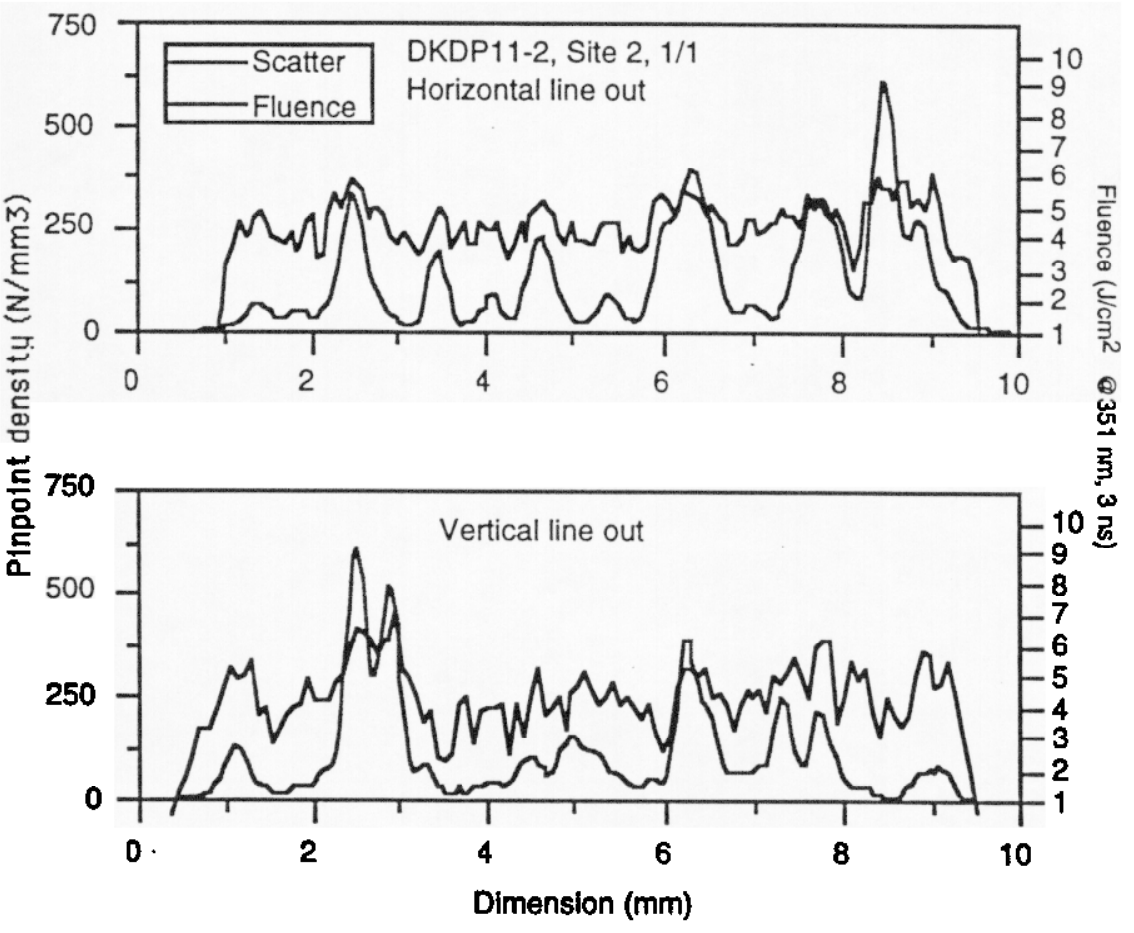

Figure 7. Illustration of scatter map and spatial profile analysis procedure. Lineouts through commonly identifiable features in both maps were taken but areas of scatter diagnostic saturation were avoided. The fluence lineouts were length scaled to match scatter peaks. This allows a direct comparison of pinpoint density to local fluence in the beam.

\subsection{Results and analysis}

The results of the lineout analysis on samples DKDP11, 214 and LL6-56 are shown for $1 / 1$ shots in Figure 8 . 


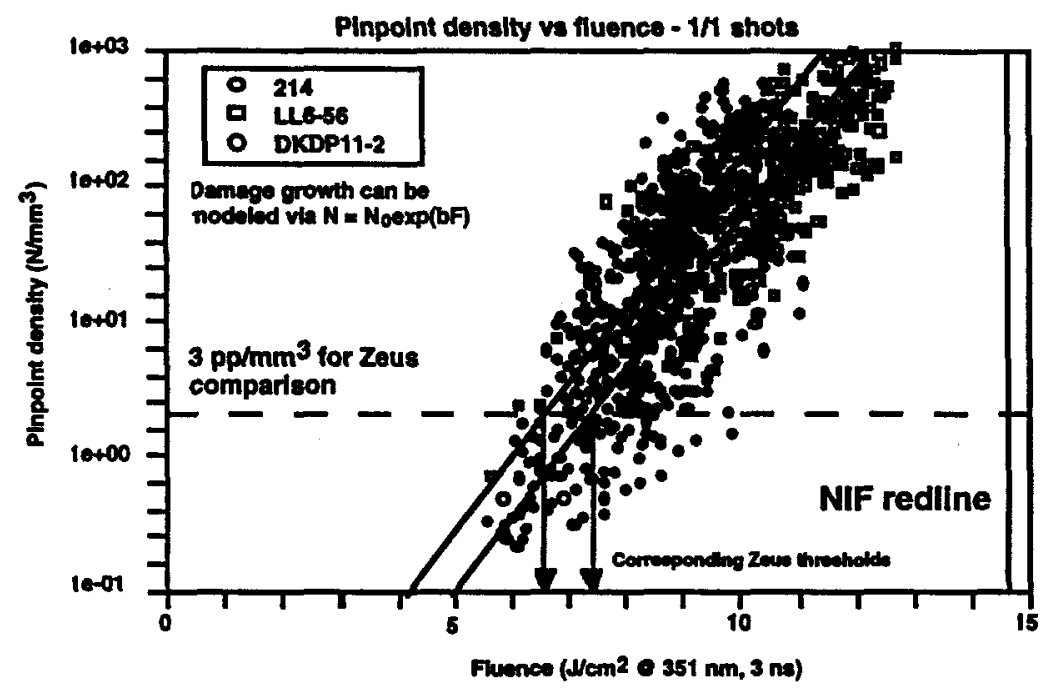

Figure 8. Damage density vs. fluence for $1 / 1$ shots on DKDP11,214 and LL6-56. The arrows indicating the corresponding ZEUS thresholds are predictions based on the detection threshold for its damage diagnostic of $3 \mathrm{pp} / \mathrm{mm}^{3}$. In a normal automated test, ZEUS does not measure unconditioned thresholds.

The results of Figure 8 show several features. First, damage growth appears to be exponential and can be fit using $\mathrm{N}(\mathrm{F})=\mathrm{N}_{\mathrm{o}} \exp (\mathrm{bF})$. As seen in Figures 9 and 10 this holds for all data. Fit data $\left(\mathrm{N}_{\mathrm{o}}\right.$ and $\left.\mathrm{b}\right)$ of these curves is given in Table 3. The data of Table 3 will have large error bars (not shown) and should not be taken too literally, however, due to the large spread in the data. Second, this evolution will lead to pinpoint densities in excess of $10^{4} \mathrm{pp} / \mathrm{mm}^{3}$ at the NIF redline fluence (14.3 J/cm $\mathrm{cm}^{2}$ for $3 \omega, 3 \mathrm{~ns}$ pulses). Lastly, the fluences corresponding to the $3 \mathrm{pp} / \mathrm{mm}^{3}$ sensitivity of the ZEUS ADT are 6.6 $\mathrm{J} / \mathrm{cm}^{2}$ for DKDP1 1 and $7.4 \mathrm{~J} / \mathrm{cm}^{2}$ for both LL6-56 and 214.

Figure 9 shows the damage evolution of sample 214 as a function of ramped exposure sequences given in Figure 4.

Sample 214 Pinpoint density ve nuence

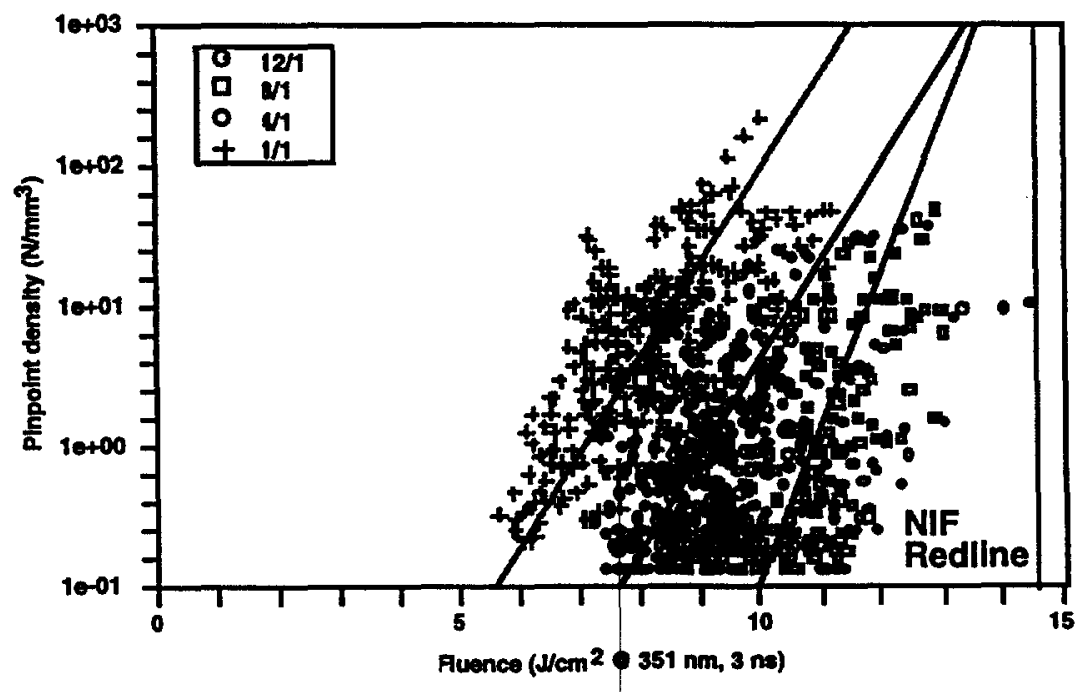

Figure 9. Damage evolution of sample 214 as a result of laser conditioning shot sequences.

Several things are evident from the damage density plot for sample 214. The exponential damage growth repeats for all ramped sequences, however, the slope increases for the conditioned sequences. Second, the increase in damage threshold 
appears to stop between the $8 / 1$ and $12 / 1$ shot sequences while the damage density continues to drop between $8 / 1$ and $12 / 1$ ramps in agreement with Figure 5. Third, the intersection of the 8/1 -12/1 exponential line with the NIF redline indicates that there will be levels of damage on the order of $10^{4} \mathrm{pp} / \mathrm{mm}^{3}$, comparable to the $1 / 1$ case in the crystal at that fluence. To acertain how the samples performed we compared the conditioned performance using $8 / 1$ shot data. The results are shown in Figure 10.

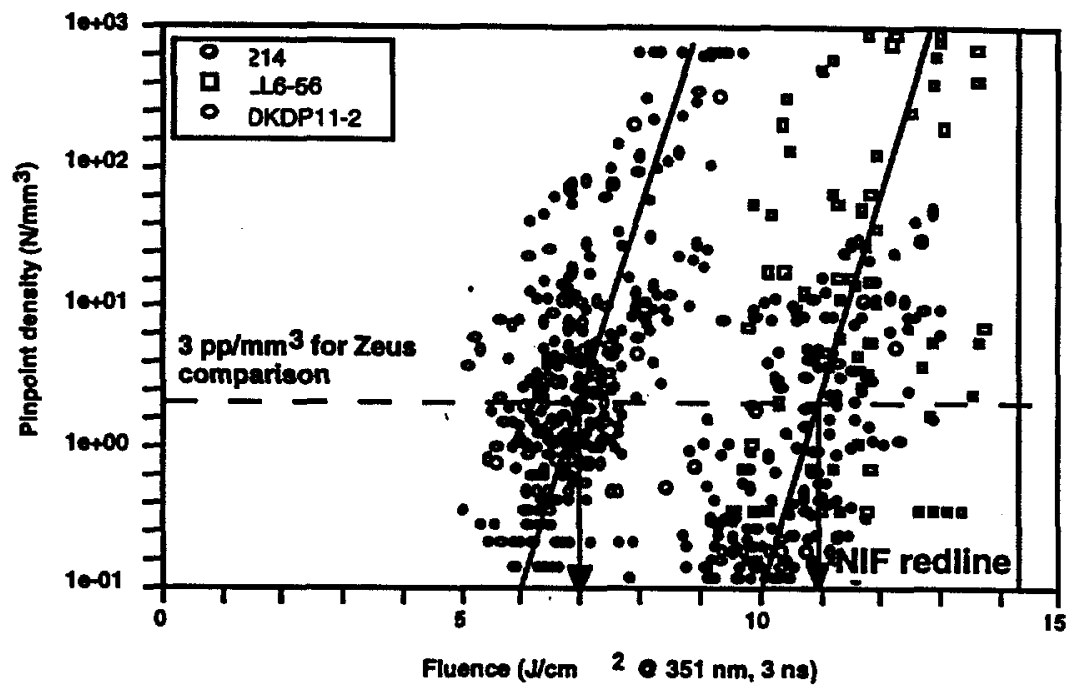

Figure 10. Damage evolution plot of 8/1 ramped exposure sequences for samples DKDP11, 214 and LL6-56. The plot shows a 1.4X increase in the damage threshold for samples 214 and LL6-56 and only a 1.1X increase for samples DKDP11.

This figure shows the same exponential damage behavior as evident in previous figures. In particular DKDP11 shows only a $1.1 \mathrm{X}$ increase in the $3 \mathrm{pp} / \mathrm{mm}^{3}$ fluence over the $1 / 1$ case, but exhibits an increase in the slope of damage evolution from 1.5 to 2.5. Samples 214 and LL6-56 exhibit essentially identical damage behavior, showing a conditioned $3 \mathrm{pp} / \mathrm{mm}^{3}$ threshold of $11 \mathrm{~J} / \mathrm{cm}^{2}$ for an overall threshold increase of $1.4 \mathrm{X}$. The slopes of the conditioned damage evolution curves for samples 214 and LL6-56 are the same (2.0) while DKDP11 has a slope of 2.5.

Table 3. Fit parameters for $1 / 1$ and $8 / 1$ shot sequences for samples 214 , LL6-56 and DKDP11. Error bars (not given) on the fit parameters are large due to the spread in the data.

\begin{tabular}{|c|c|c|c|}
\hline Sample & Ramp sequence & $\mathbf{N}_{0}$ & b \\
\hline \multirow{2}{*}{214} & $1 / 1$ & $3 \times 10^{-6}$ & 1.75 \\
\cline { 2 - 4 } & $7 / 1$ & $5 \times 10^{-10}$ & 2.0 \\
\hline \multirow{2}{*}{ LL6-56 } & $1 / 1$ & $1.5 \times 10^{-5}$ & 1.5 \\
\cline { 2 - 4 } & $8 / 1$ & $5 \times 10^{-10}$ & 2.0 \\
\hline \multirow{2}{*}{ DKDP11 } & $1 / 1$ & $7.5 \times 10^{-5}$ & 1.5 \\
\cline { 2 - 4 } & $8 / 1$ & $10^{-7}$ & 2.5 \\
\hline
\end{tabular}

\subsection{Total damage in the crystals}

To quantify the overall level of damage at each test site we performed a simple conversion of damage density to total number of pinpoints in the irradiated volume. Each volume element was considered to be $0.05 \times 0.05 \times 10 \mathrm{~mm}^{3}$ corresponding to the step size of the raster scan and a $1 \mathrm{~cm}$ thick test sample. When the number of pinpoints in each volume element was determined the total was calculated by summing over the entire irradiated volume (i.e. scatter map). This approach is the simplest possible, and neglects effects of the fluence distribution and resulting nonuniform damage density. Nevertheless the results shown in Table 4 are quite dramatic and point out the reduction in overall damage level possible by laser conditioning. Sample LL6-56 shows the highest level of pinpoint reduction due to conditioning with a 300X drop between the $1 / 1$ and 8/1 
cases. This is followed by sample 214 with a reduction factor of 42 between the $1 / 1$ and $12 / 1$ cases. DKDP11 shows the least amount of pinpoint reduction (11:1) between the $1 / 1$ and $8 / 1$ cases.

Table 4. Total number of pinpoints generated at each test site.

\begin{tabular}{|c|c|c|c|}
\hline Sample & Sequence (shot/site) & Number of pinpoints $(\mathbb{N})$ & $\Delta \mathbf{N}\left(\mathbf{N} / \mathbb{N}_{\min }\right)$ \\
\hline \hline \multirow{2}{*}{ LL6-56 } & $1 / 1$ & 220194 & $300: 1$ \\
\cline { 2 - 4 } & $8 / 1$ & 736 & $1: 1$ \\
\hline \multirow{2}{*}{ DKDP11 } & $1 / 1$ & 75380 & $11: 1$ \\
\cline { 2 - 4 } & & 6713 & $1: 1$ \\
\hline \multirow{2}{*}{ KDP 214 } & $1 / 1$ & 5458 & $42: 1$ \\
\cline { 2 - 4 } & $4 / 1$ & 1524 & $12: 1$ \\
\cline { 2 - 4 } & $7 / 1$ & 531 & $4: 1$ \\
\cline { 2 - 4 } & $12 / 1$ & 129 & $1: 1$ \\
\cline { 2 - 4 } & & &
\end{tabular}

\subsection{Comparison of OSL and ZEUS test results.}

In order to compare the test results between OSL and ZEUS we converted the $8 / 1,3 \mathrm{pp} / \mathrm{mm}^{3} \mathrm{ZEUS}$ threshold equivalents to $7.6 \mathrm{~ns}$ pulse duration using $\tau^{0.5}$ scaling. These OSL thresholds were then plotted on the appropriate ZEUS ADT damage probability curves. The results are shown in Figure 11.

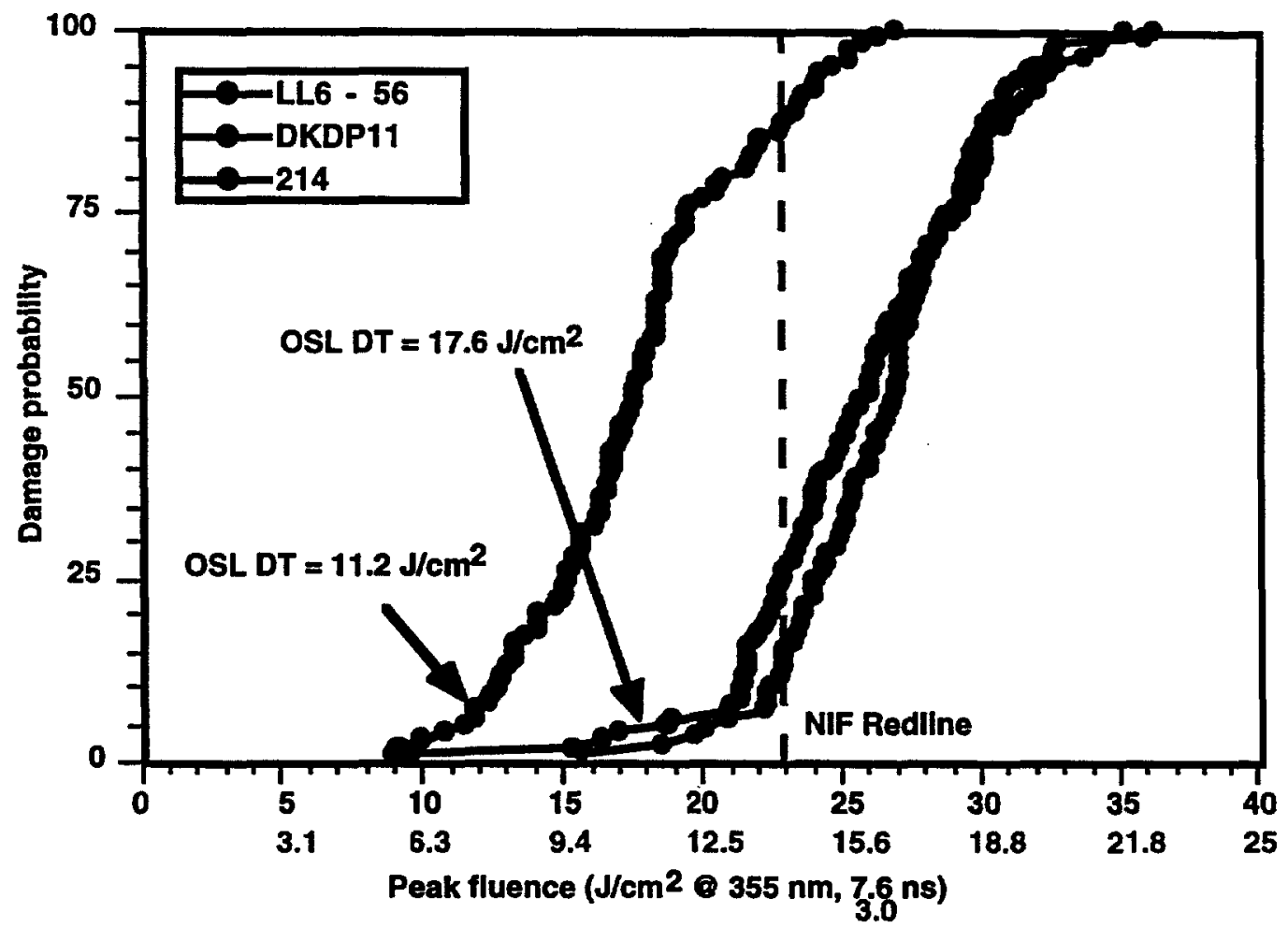

Figure 11. Comparison of OSL and ZEUS ADT test results. The OSL $3 \mathrm{pp} / \mathrm{mm}^{3}$ fluences for each sample are shown on the corresponding damage probability curve and fall on the toe of both curves. The relative performance of the sample set is the same on OSL and ZEUS. LL6-56 and 214 exhibit the same damage behavior while DKDP11 is $-1.5 \mathrm{X}$ lower, as seen in OSL testing. 


\section{DISCUSSION OF RESULTS}

\subsection{General comments}

The test results of the preceding sections have shown the effect of laser conditioning on the damage performance of a variety of KDP and KD*P samples relevant to the NIF project. Several conclusions can be drawn from the data. First, for all samples tested, 8 shot ramps of equal fluence interval appear to provide the maximum attainable increase in damage threshold. The increase in threshold appears to saturate between the 8/1 and 12/1 cases for sample 214, and presumably for LL6-56 as well.

The pinpoint density evolution can be modeled by the exponential function $N=N_{0} \exp (b F)$. Damage density evolution after onset is more severe for conditioned ramps as indicated by the steeper slopes of the fit lines for all samples tested. But the overall level of damage for conditioned sites is less due to the lower $\mathrm{N}_{\mathrm{o}}$ values of the conditioned sequences. This was confirmed in Figure 5 and by the results of Table 4. Samples 214 and LL6-56 showed the greatest level of conditioning, exhibiting a 1.4X increase in damage threshold and up to $300 \mathrm{X}$ pinpoint reduction. Sample DKDP11 exhibited the lowest overall damage performance with only a $1.1 \mathrm{X}$ increase in damage threshold. This is reflected in the highest $\mathrm{N}_{\mathrm{o}}$ value $(7.5 \mathrm{X}$ $10^{-5} \mathrm{~cm}^{-3}$ ) for $1 / 1$ sites and the steepest slope (2.5) for $8 / 1$ curves.

The behavior of the samples on OSL mimics the behavior measured on ZEUS. Specifically we have measured a $1.5 \mathrm{X}$ increase in the damage threshold for samples LL6-56 and 214 on both systems. The 8/1 ZEUS threshold detection pinpoint density (3 $\left.\mathrm{pp} / \mathrm{mm}^{3}\right)$ fluences fall on the toe of the damage probability curves. These results validate using small area $(\phi \sim 1 \mathrm{~mm})$ beams for assessment of NIF THG damage.

\subsection{Implications for the NIF}

The results of these experiments prove that on-line conditioning of the NIF triplers to near NIF redline fluences is feasible. Because of the inconclusive nature of previous raster conditioning studies on $K D^{*} \mathrm{P}$, on-line conditioning is currently the only proven method for increasing the laser damage performance of $K D^{*} \mathrm{P}$. Despite this there remain serious questions regarding the practicality of on-line conditioning. We discuss a number of these questions in the following section.

We address first the question of what happens in the crystal when laser damage does occur. Despite laser conditioning, the damage density plots indicate that there will be substantial pinpoint formation when fluences approach the NIF redline (14.3 $\mathrm{J} / \mathrm{cm}^{2}$ ). Here extrapolated pinpoint densities for each sample are on the order of $10^{4} \mathrm{pp} / \mathrm{mm}^{3}$. To understand whether this level of pinpoint density will lead to generation of large levels of damage, it is imperative to know the predicted beam statistics of a full fluence NIF pulse. Mapping the damage density evolution equations onto a model NIF spatial profile will allow calculation of the expected number of pinpoints to be generated in the crystal. Currently a number of model NIF profiles have been obtained and this analysis is underway. We also hope to use this information to understand the apparent paradox between the lack of damage on the Beamlet $37 \mathrm{~cm}$ tripler and the high fluence shots it has seen. Furthermore, this modeling will give us an understanding of the overall survivability of the NIF triplers.

High levels of damage are expected to adversely affect local frequency conversion. To answer questions regarding conversion efficiency, a modeling and experimental program needs to be undertaken. A model is needed which incorporates the damage density evolution data from these experiments. To first order, the damage pinpoints could be considered as obscurations in the beam which do not allow conversion. Further refinements of the model would consider the effects of diffraction on the conversion process. Such a modeling effort is not now under consideration, but should be easily incorporated into existing frequency conversion modeling codes. On the other hand, the effect of damage on frequency conversion is scheduled to be investigated in the coming months. The effort will consist of installing a DKDP11 tripler on OSL, optimizing the conversion efficiency and then increasing incident power until damage is observed. This experiment will allow us to investigate whether there are changes to the damage mechanism as a result of the mixing process, and will also be the first time a controlled, in-situ, multiwavelength damage test has been conducted on $K D^{*} \mathrm{P}$ at LLNL.

Questions regarding the long term stability of damage are also an issue, albeit a secondary one. Observations from Zeus testing indicate that once a pinpoint forms it remains stable even when exposed to succeeding shots of higher fluence. As 
fluence increases, however, higher numbers of pinpoints are generated locally. In the case of poor samples, or very high fluence the number of pinpoints generated will ultimately be so great that the bulk mechanical properties of the sample are altered and propagating catastrophic damage is the result. It seems unlikely that this stage of damage would be reached on NIF before its impact on frequency conversion is noticed or it is discovered by inspection. However, this issue will also be investigated in on-going damage tests on Zeus.

An additional question is understanding how shifts in the spatial fluence distribution will affect the damage performance of an on-line conditioned tripler. Because of the spatial distribution of the beam fluence, each portion of the crystal will see a different conditioning ramp. If the spatial profile changes significantly for some reason, say going from the 3 ns NIF pulse to a 1 ns pulse, there will be the possibility of damage caused by shifts of high fluence portions of the beam onto less conditioned portions of the tripler. It should be possible to model the expected behavior of such a situation using the damage evolution curves and threshold information generated in this OSL campaign.

Probably of greater concern than shifts in the beam fluence distribution are shifts in the overall alignment of the beam through the tripler itself. Not only will this lead to shifts in the beam fluence over conditioned areas, it will likely expose unconditioned parts of the tripler to high fluence, almost certainly leading to damage at the edges of the beam. Currently there is no simple way to address this problem with the on-line conditioning process.

\subsection{Comments regarding off-line conditioning methods}

The discussion of 4.2 points out the deficiencies of on-line conditioning. Despite the feasibility shown in these experiments there remains significant concerns which limit the attractiveness of the process. Hence there is a strong desire to explore alternative, off-line conditioning methods. The advantages of such a process are numerous. Off-line conditioning will make it possible to uniformly condition an entire tripler to a known degree, beyond the clear aperture thus eliminating concerns about damage due to beam fluence and alignment shifts. It will also be significantly less costly and will not impact NIF shot schedules.

LLNL has explored the conditioning problem of $\mathrm{KDP} / \mathrm{KD} * \mathrm{P}$ in some detail concentrating on mainly on thermal annealing and $1 \omega$ laser conditioning studies ${ }^{1-4}$. These techniques are promising at $1064 \mathrm{~nm}$ but are not effective at conditioning at $3 \omega$. The results of this OSL campaign indicate that $3 \omega$ raster scanning should be revisited. It is difficult to envision that a raster conditioning process is significantly different than the exposure sequences performed in this work. For this reason, and because we have improved damage detection diagnostics than those available to Rainer and Staggs ${ }^{5}$ it should be possible to arrive at a definite conclusion regarding raster scanning at $3 \omega$. This work is currently planned for the coming fiscal year.

Another possible method of off-line conditioning involves exposure of the samples to broadband, low fluence UV radiation for long periods of time. This technique has not been investigated before and is much more speculative than raster conditioning. Samples could be exposed to pulsed UV sources such as flashlamps, or for longer durations to low power sources such as UV solar simulators. There are currently no short-term plans to pursue this technology, however, a small scale feasibility study would be straightforward.

\section{CONCLUSION}

We have performed ramped fluence experiments on a variety of KDP and $K D * P$ samples relevant to the NIF laser project to investigate phenomenological effects of $3 \omega$ laser conditioning. New diagnostics and data analysis techniques have been developed to measure the damage density at a test site and correlate it to local fluence in the beam.

We have shown in this work that on-line conditioning of NIF KD*P tripler crystals is feasible. An $8 / 1$ exposure sequence starting from approximately $1 \mathrm{~J} / \mathrm{cm}^{2}$ up to fluences near the NIF redline will lead to maximum conditioning. This conditioning sequence will lead to approximately a $1.5 \mathrm{X}$ increase in the damage threshold and a overall damage reduction of up to $300 \mathrm{X}$. The damage thresholds measured on OSL are in agreement with small area tests performed on the Zeus automated damage test facility.

These experiments also led to the discovery that damage density evolution in $\mathrm{KDP} / \mathrm{KD}^{*} \mathrm{P}$ is exponential and can be fit using $\mathrm{N}=\mathrm{N}_{\mathrm{o}} \exp (\mathrm{bF})$ to obtain an initial defect density $\left(\mathrm{N}_{0}\right)$ and slope $(b)$. 
Despite the feasibility of the on-line conditioning technique there are also deficiencies. In particular there are significant concerns regarding damage occurring due to beam misalignment exposing an unconditioned area. The cost of conditioning 192 triplers on-line also limits the attractiveness of this technology and makes off-line conditioning methods much more desirable. The state-of-the-art in alternative off-line conditioning techniques was discussed and a reevaluation of $3 \omega$ raster scanning techniques was called for.

We have discussed the possible impacts of laser damage on the NIF triplers at levels up to $10^{4} \mathrm{pp} / \mathrm{mm}^{3}$. It is expected that damage at this level will have a significant impact on frequency conversion efficiency and possibly the propagation of hot spots in the beam. To determine if this will be an issue a program of modeling and experiments has been outlined. Some elements discussed are currently underway or will be addressed in the near future. In particular the OSL damage evolution models are being applied to predicted NIF $3 \omega$ spatial profiles. A campaign for taking a rapidly grown KD*P tripler to damage during the frequency conversion process is also planned. This will allow practical limits on the damage density to be established and will also serve as a baseline for modeling codes.

This work performed under the auspices of the U. S. Department of Energy by Lawrence Livermore National Laboratory under Contract No. W-7405-ENG-48.

\section{REFERENCES}

1. F. Rainer, L.J. Atherton, J.J. DeYoreo, "Laser damage to production- and research grade KDP crystals," in Laser Induced Damage in Optical Materials. SPIE Proceedings, Volume 1848, edited by H.E. Bennet et. al., 46-58, SPIE, Bellingham, WA. 1992

2. F. Rainer et. al., "A historical perspective on fifteen years of laser damage thresholds at LLNL," in Laser Induced Damage in Optical Materials. SPIE Proceedings, Volume 2114, edited by H.E. Bennet et. al., 9-22, SPIE, Bellingham, WA. 1993

3. J. Swain, S. Stokowski, D. Milam, F. Rainer, "Improving the bulk laser damage resistance of potassium dihydrogen phosphate by pulsed laser irradiation," Applied Physics Letters 40(4), pp 350-352, 1982.

4. J. Swain, S. Stokowski, D. Milam, "The effect of baking and pulsed laser irradiation on the bulk laser threshold of potassium dihydrogen phosphate crystals," Applied Physics Letters 41(1), pp 12-14, 1982.

5. F. Rainer, M. Staggs, C. Barker, F. DeMarco, M. Kozlowski, LDG 93-004 (LLNL Internal Memo): "Status of large-area raster conditioning of $\mathrm{KDP}, " 1 / 25 / 1993$.

6. Y. Sun, C. Li, H. Gong, "Laser treatment to improve the ability to resist laser damage in potassium dihydrogen phosphate crystals," in Laser Induced Damage in Optical Materials, SPIE Proceedings, Volume 1848, pp 574-577, 1992.

7. M. Runkel, M. Yan, J. DeYoreo, N. Zaitseva, "The effect of impurities and stress on the damage distributions of rapidly grown KDP crystals," in Laser Induced Damage in Optical Materials. SPIE Proceedings, 1997 


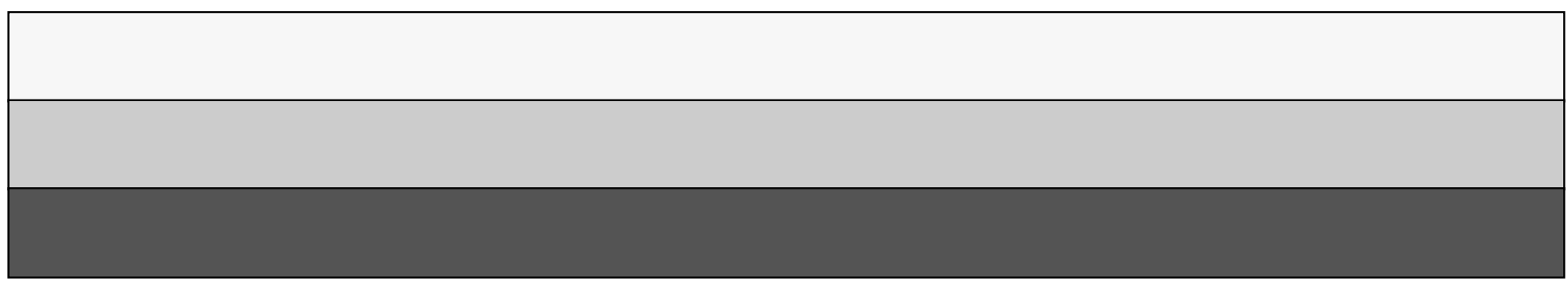

